\title{
Estratégias auxiliares para graduação dos tumores astrocíticos segundo os critérios histopatológicos estabelecidos pela OMS
}

\section{Auxiliary strategies for astrocytic tumor graduation according to histopathological criteria established by WHO}

Mário Henrique Girão Faria'; Régia Maria do Socorro Vidal do Patrocínio²; Silvia Helena Barem Rabenhorst ${ }^{3}$

Unitermos
Astrocitomas
Histopatologia
Inteligência artificial
OMS

OMS

\section{resumo}

Introdução: Apesar das recentes descobertas acerca das alterações moleculares envolvidas na tumorigênese dos astrocitomas, a análise histológica convencional permanece o melhor método diagnóstico e razoável indicador prognóstico dos tumores astrocíticos. Todavia, a rotina histopatológica preconizada pela Organização Mundial da Saúde (OMS) mostra-se laboriosa, sendo marcada pela baixa reprodutibilidade. Objetivo: O presente estudo objetivou desenvolver estratégias auxiliares para graduação dos tumores astrocíticos a partir dos critérios histológicos estabelecidos pela OMS. Material e método: Procedeu-se à análise clínico-epidemiológica e à reavaliação histopatológica de 55 astrocitomas de diferentes gradações (13 grau I, 14 grau II, sete grau III e 21 grau IV) e de cinco amostras de tecido cerebral não-tumoral (grupo controle). Resultados: A distribuição por idade, por sexo e pela localização tumoral dos portadores dessas neoplasias reproduziu, de um modo geral, as tendências mundiais. A presença de células gigantes multinucleadas e de gemistócitos foi associada à malignidade tumoral. Os achados histopatológicos avaliados segundo critérios semiquantitativos e pelo modelo de aprendizagem CART confirmaram a gradação original, reiterando a aplicabilidade e a reprodutibilidade dos mesmos. Conclusão: Tais resultados indicaram a árvore de decisão CART e o somatório de escores semiquantitativos como estratégias práticas e auxiliares para a graduação dos astrocitomas segundo os critérios da OMS. abstract

Background: Despite recent discoveries concerning molecular alterations involved in astrocytoma tumorigenesis, the conventional histological analysis remains the best diagnostic method and a reasonable prognostic indicator for astrocytic tumors. However, the histopathological routine recommended by the World Health Organization (WHO) is laborious and marked by low reproducibility. Objective: The present study aimed to develop auxiliary strategies for astrocytic tumor graduation according to histological criteria established by WHO. Material and method: A clinical and epidemiological analysis and a histopathological evaluation were performed in 55 astrocytomas of different graduations (13 grade I, 14 grade II, seven grade III, 21 grade IV) and five samples of non-tumor tissue (control group). Results: The distribution by age, sex, and tumor localization of astrocytomas in patients reproduced, in a general way, worldwide trends. The presence of multinucleated cells and gemistocytes was associated with tumoral malignancy. The histopathological findings evaluated through semi-quantitative criteria and CART modeling confirmed the primary classification, reaffirming their applicability and reproducibility. Conclusion: These results indicate the CART decision tree and the totality of semi-quantitative scores as practical and auxiliary strategies for astrocytoma graduation as stated by WHO criteria.

1. Médico; mestre em Farmacologia; pesquisador do Laboratório de Genética Molecular (LABCEM) da Universidade Federal do Ceará (UFC).

2. Médica patologista do Departamento de Patologia e Medicina Legal da Faculdade de Medicina da UFC e Biomédica, Pesquisas e Serviços Ltda. (BIOPSEE); membro da Sociedade Brasileira de Patologia (SBP).

3. Bióloga; doutora em Cenética; professora-adjunta IV do Departamento de Patologia e Medicina Legal da Faculdade de Medicina/UFC; coordenadora do LABCEM/UFC. Artigo baseado na dissertação de mestrado Estudo imuno-histoquímico das alteraç̧ês moleculares nos tumores astrociticos: vias tumorigênicas e indicadores de resistência, defendida na Faculdade de Medicina/UFC, em 2005. 


\section{Introdução}

Os astrocitomas constituem o principal tipo histológico entre os tumores primários do sistema nervoso central (SNC), configurando $1,32 \%$ de todas as neoplasias diagnosticadas anualmente ${ }^{(6)}$. A despeito da baixa incidência, os astrocitomas representam os mais freqüentes tumores sólidos da infância e a segunda causa de mortalidade por câncer nessa faixa etária, ficando atrás somente das leucemias. Nos adultos, a sobrevida média após cinco anos do diagnóstico desses tumores é de $32 \%$, constituindo a terceira e a quarta causa de mortalidade por câncer entre homens e mulheres, respectivamente ${ }^{(21)}$.

O termo astrocitoma foi cunhado por Virchow para designar os tumores compostos predominantemente por astrócitos atípicos (núcleos aumentados, alongados ou hipercromáticos com citoplasmas escassos, poucos definidos) ${ }^{(26)}$, porém só foi empregado definitivamente na classificação proposta por Bailey e Cushing ${ }^{(1)}$. Na classificação histopatológica da Organização Mundial da Saúde (OMS) para os tumores próprios do SNC, admite-se que as diversas apresentações histológicas dos astrocitomas possam ser divididas em diferentes graus de malignidade, variando de I a IV(15). Em grande parte, essa graduação resulta do reconhecimento de indicadores de anaplasia (atipia nuclear, pleomorfismo, atividade mitótica, hiperplasia endotelial e necrose) típicos de cada variante tumoral através da análise histológica rotineira por microscopia óptica. Como regra geral, o grau tumoral é baseado nas áreas de maior atipia, assumindo que essa população de células é a que determina o curso da doença ${ }^{(23,24)}$. Além de manifestar o comportamento biológico tumoral, permitindo inferências prognósticas, o acúmulo de achados anaplásicos parece refletir a progressão das alterações moleculares adquiridas durante o processo de transformação neoplásica ${ }^{(10,14)}$.

No esquema de graduação adotado pela OMS, a presença de notória atividade mitótica define os tumores de alto grau (III e IV). A presença de evidente necrose tumoral, acompanhada ou não por hiperplasia endotelial, delimita o grau IV (glioblastoma). A detecção unicamente de atipia citológica (pleomorfismo celular e/ou atipia nuclear) estabelece o grau II. Os tumores do grau I (predominantemente astrocitomas pilocíticos) consistem em entidades distintas: além da ausência de anaplasia franca têm sua gradação sugerida por dados clínicos (idade do paciente, localização tumoral, aspecto radiológico) ${ }^{(10,27)}$.

A despeito da aparente simplicidade na aplicação dos critérios histológicos de graduação e das notórias particula- ridades epidemiológicas e radiológicas observadas entre as diferentes gradações dos astrocitomas, a rotina diagnóstica histopatológica permanece laboriosa, sendo marcada pela baixa reprodutibilidade interobservador $(3,7,12,19,20,22,24,25)$. Mesmo assim, a análise histológica convencional permanece o melhor método diagnóstico e razoável indicador prognóstico para os tumores astrocíticos ${ }^{(3,11,19)}$. Tal fato deve-se à relativa carência de correlações clínicas diretas entre as alterações genéticas implicadas na tumorigênese dos astrocitomas e o comportamento biológico desses tumores, especialmente na definição de conjecturas prognósticas(7, 13, 24).

Nesse contexto, o presente estudo objetivou desenvolver estratégias auxiliares para graduação dos tumores astrocíticos a partir dos achados histopatológicos estabelecidos como critérios de gradação pela OMS.

\section{Material e método}

\section{Casuística}

Realizou-se levantamento dos tumores astrocíticos humanos fixados em formalina e incluídos em parafina provenientes do arquivo do Laboratório Biomédica, Pesquisas e Serviços Ltda. (BIOPSE ${ }^{\circledR}$ ) referentes aos exames histopatológicos rotineiros realizados no período entre 1999 e 2003. Admitiram-se como critérios de inclusão a existência de mais de uma amostra (bloco) para cada caso, o bom estado de conservação dos blocos e a adequação da graduação histológica utilizada quando do diagnóstico em relação à padronizada pela $\mathrm{OMS}^{(14)}$. Dessa forma, 55 astrocitomas foram selecionados: 13 grau I, 14 grau II, sete grau III e 21 grau IV, configurando uma amostragem não-probabilística.

Como referência de normalidade, cinco amostras de tecido cerebral não-tumoral fixadas em formalina e incluídas em parafina foram obtidas do material de rotina do Setor de Necropsia do Departamento de Patologia e Medicina Legal da Faculdade de Medicina da Universidade Federal do Ceará (UFC). Foram selecionados casos em que a causa imediata da morte e a doença de base em nada remeteram à presença de neoplasia intra ou extracerebral.

A coleta das amostras foi autorizada pelos responsáveis de cada instituição, sendo o projeto de pesquisa aprovado pelo comitê de ética em pesquisa do complexo hospitalar da UFC sob protocolo 32/04, dentro das normas que regulamentam as pesquisas em seres humanos, segundo a 
Resolução no 196/96 do Conselho Nacional de Saúde - Ministério da Saúde/Brasil.

\section{Preparo das lâminas e análise histopatológica}

Procedeu-se à manufatura de cortes histológicos dos blocos selecionados a $5 \mu \mathrm{m}$ seguida de coloração por hematoxilina/eosina. As lâminas foram avaliadas por três examinadores experientes de forma independente: após a confirmação do diagnóstico histológico (variante e graduação, segundo critérios da OMS), realizou-se análise dos achados histopatológicos específicos (atipia nuclear, pleomorfismo celular, hiperplasia endotelial, presença de necrose, índice mitótico, celularidade, presença de gemistócitos e existência de células gigantes) em dez campos de maior representatividade tumoral utilizando magnificação de 100-400x, sendo atribuídos escores semiquantitativos conforme previamente estabelecido (Tabela 1). Os eventuais resultados conflitantes foram revisados pelo conjunto dos examinadores para definição consensual da análise.

\section{Análise estatística}

Os escores referentes às análises histopatológicas, bem como os dados acerca da localização tumoral, da idade e do sexo dos pacientes, foram tabulados utilizando-se o programa Microsoft Excel ${ }^{\circledR}$ 2000. Empregou-se o programa
SPSS ${ }^{\circledR} 13.0$ para comparação dos dados através de testes não-paramétricos (teste de Shapiro-Wilk, teste $H$ de KruskalWallis e teste $U$ de Mann-Whitney), sendo os resultados expressos como média \pm desvios padrões da média (DPM). Foram considerados significativos valores de $p<0,05$.

Aplicou-se também a metodologia classification and regression trees $(\mathrm{CART})^{(2)}$ de aprendizagem automática (inteligência artificial) para construção de árvores de decisão com complexidade limitada através do programa CART 5.0. Árvores de decisão (Figura 1) são estruturas que se assemelham a árvores invertidas, nas quais as folhas representam as conclusões (diagnósticos), os nós expressam as variáveis (sinais, sintomas, achados) e os ramos distinguem os valores assumidos pelas variáveis ${ }^{(17)}$. O primeiro nó é denominado raiz.

O CART inicia sua abordagem gerando uma árvore de tamanho máximo a partir dos dados descritos na casuística original. Assim, cada caminho que segue da raiz às folhas representaria um único caso. Posteriormente, procede-se à poda dos ramos, gerando árvores cada vez menores, até a escolha da melhor estratégia de decisão através de múltiplos processos estatísticos ${ }^{(5)}$.

Quando a quantidade de casos disponíveis para a construção da árvore é pequena, não possibilitando a divisão da amostra em um conjunto de aprendizagem e outro conjunto teste, o CART emprega a validação cruzada. Esse

\section{Tabela 1 Significado dos escores utilizados para análise dos achados histopatológicos}

\begin{tabular}{lcc}
\hline Achados & Escore & Significado \\
Atipia nuclear* (ATIPN) Pleomorfismo celular* (PLEOC) & 0 & Ausente \\
Hiperplasia endotelial* $^{*}$ (HIPEND) & 1 & Discreto(a) \\
Necrose* (NEC) & 2 & Moderado(a) \\
& 3 & Intenso(a) \\
Índice mitótico* (IM) & 0 & Ausência de figuras mitóticas \\
& 1 & $\leq 2$ mitoses/campo \\
& 2 & 3 a 4 mitoses/campo \\
& 3 & $\geq 5$ mitoses/campo \\
Celularidade (CEL) & 0 & Normal \\
& 1 & Aumento discreto \\
Gemistócitos (GEM) & 2 & Aumento evidente (células não-justapostas) \\
Células gigantes (CGIG) & 3 & Elevada (células justapostas) \\
\hline
\end{tabular}

*Critérios de graduação histopatológica dos astrocitomas segundo classificação da OMS. 


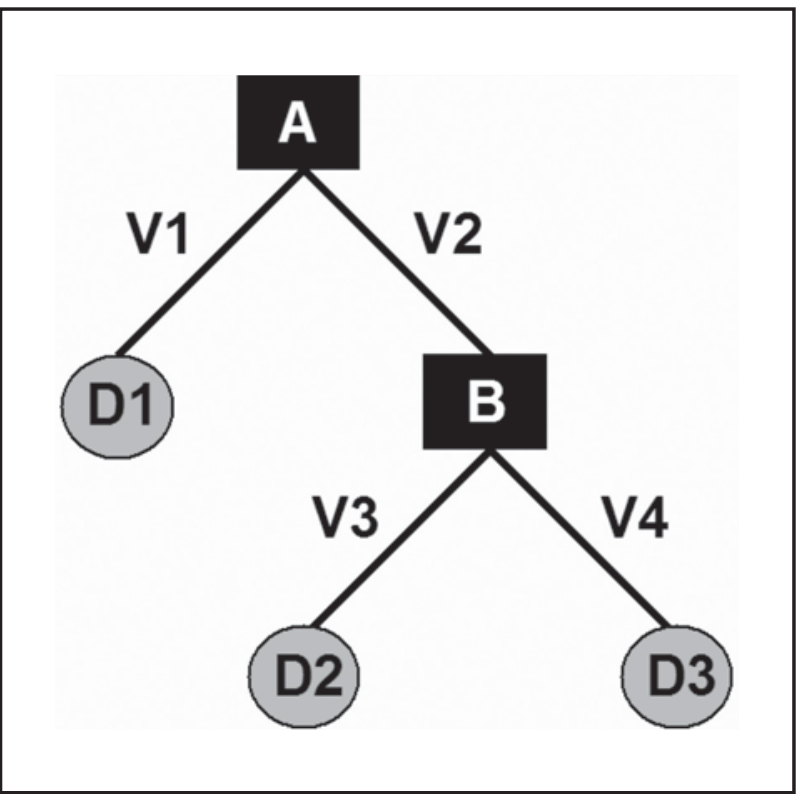

Figura 1 - Modelo esquemático de uma árvore de decisão. A e B (retângulos): nós; V1, V2, V3 e V4 (traços): ramos; D1, D2 e D3 (círculos): folhas; regras diagnósticas: $A=V 1$ implica em D1; $A=V 2$ e $B=V 3$ implicam em D2 $e A=V 2$ e $B=V 4$ implicam em D3

procedimento utiliza subamostras disjuntas de mesmo tamanho para a formatação de subárvores, utilizando o mesmo processo de desbaste. Dessa forma, estima-se o erro real contido nas sentenças de decisão a partir da comparação entre as subárvores e a árvore principal, construída com a totalidade dos dados. A associação entre a poda e a validação cruzada fornece uma árvore de decisão de complexidade reduzida e com taxas de erro real satisfatórias ${ }^{(2,5)}$.

\section{Resultados}

\section{Aspectos clínico-epidemiológicos}

A Tabela 2 apresenta em detalhes todos os resultados obtidos. A distribuição dos casos de acordo com a faixa etária dos pacientes (Figura 2) demonstrou que 83,33\% dos astrocitomas grau I acometeram pacientes jovens (4 a 19 anos), 71,43\% dos astrocitomas grau II ocorreram em indivíduos adultos (23 a 48 anos), 42,86\% dos astrocitomas grau III manifestaram-se no terceiro decênio (22 a 28 anos), ao passo que $45 \%$ dos astrocitomas grau IV surgiram na sexta década de vida. Apesar de as duas últimas gradações mostrarem predomínios específicos pelas faixas etárias descritas, observou-se que a ocorrência do grau III prolongava-se até pacientes adultos e idosos (40 a 71 anos) e o aparecimento do grau IV estendia-se desde crianças até idosos (5 a 81 anos).

A divisão por sexo dos pacientes portadores dos tumores astrocíticos avaliados indicou relação masculino/feminino de 1,89 (67\% de pacientes do sexo masculino e $33 \%$ do sexo feminino), sendo essa relação de 1,28 quando contabilizados todos os pacientes detectados no levantamento geral. Quanto à localização tumoral, prevaleceram os sítios frontal, cerebelar e parietal. A maior parte (75\%) dos tumores grau I estava situada nos hemisférios cerebelares, enquanto cerca da metade (55\%) dos tumores grau IV encontrava-se nos lobos cerebrais frontais.

Tabela 2

\section{Graduação tumoral, dados clínico-epidemiológicos gerais e escores histopatológicos referentes} aos casos estudados

\begin{tabular}{lccccccccccccc} 
Caso & \multicolumn{1}{c}{ Grau Geral } & \multicolumn{10}{c}{ Escores histopatológicos } \\
& (OMS) & Idade & Sexo & Local & CEL & CGIG & GEM & PLEOC* & ATIPN* & IM* & NEC* & HIPEND* & Soma \\
NT01 & NT & 48 & M & Parietal & 0 & 0 & 0 & 0 & 0 & 0 & 0 & 0 & 0 \\
NT02 & NT & 14 & M & Parietal & 0 & 0 & 0 & 0 & 0 & 0 & 0 & 0 & 0 \\
NT03 & NT & 55 & F & Parietal & 0 & 0 & 0 & 0 & 0 & 0 & 0 & 0 & 0 \\
NT04 & NT & 15 & F & Parietal & 0 & 0 & 0 & 0 & 0 & 0 & 0 & 0 & 0 \\
NT05 & NT & 57 & F & Parietal & 0 & 0 & 0 & 0 & 0 & 0 & 0 & 0 & 0 \\
T01 & I & 10 & M & - & 2 & 0 & 1 & 1 & 1 & 0 & 0 & 0 & 2 \\
T02 & I & 19 & M & - & 2 & 0 & 0 & 0 & 0 & 0 & 0 & 0 & 0 \\
T03 & I & 34 & F & - & 1 & 0 & 0 & 0 & 0 & 0 & 0 & 0 & 0 \\
T04 & I & 6 & M & Cerebelar & 1 & 0 & 0 & 0 & 0 & 0 & 0 & 1 & 1 \\
T05 & I & 17 & F & Ventricular & 2 & 0 & 0 & 1 & 1 & 0 & 0 & 0 & 2 \\
T06 & I & 4 & F & Cerebelar & 2 & 0 & 0 & 0 & 1 & 1 & 1 & 3 & 6 \\
T07 & I & 16 & M & Cerebelar & 1 & 0 & 0 & 1 & 1 & 1 & 0 & 2 & 5
\end{tabular}




\begin{tabular}{|c|c|c|c|c|c|c|c|c|c|c|c|}
\hline T08 & 4 & $\mathrm{~F}$ & Selar & 2 & 0 & 0 & 1 & 0 & 0 & 0 & 1 \\
\hline T09 & 14 & M & Cerebelar & 2 & 0 & 0 & 0 & 1 & 0 & 0 & 2 \\
\hline T10 & 3 & M & - & 1 & 0 & 0 & 0 & 1 & 0 & 0 & 1 \\
\hline T11 & - & $\mathrm{F}$ & - & 2 & 0 & 1 & 0 & 0 & 0 & 0 & 0 \\
\hline T12 & 42 & $\mathrm{M}$ & Cerebelar & 1 & 0 & 0 & 1 & 1 & 0 & 0 & 1 \\
\hline T13 & 13 & $\mathrm{~F}$ & Cerebelar & 2 & 0 & 1 & 1 & 1 & 0 & 2 & 1 \\
\hline T14 & 56 & $\mathrm{M}$ & Cerebelar & 2 & 0 & 0 & 1 & 2 & 0 & 0 & 0 \\
\hline T15 & 34 & $\mathrm{~F}$ & - & 2 & 0 & 2 & 2 & 2 & 0 & 0 & 1 \\
\hline T16 & 23 & M & Temporal & 1 & 0 & 0 & 2 & 3 & 1 & 0 & 1 \\
\hline T17 & 36 & M & Parietal & 3 & 0 & 0 & 2 & 3 & 0 & 1 & 1 \\
\hline T18 & 47 & M & Frontal & 2 & 0 & 0 & 2 & 2 & 0 & 0 & 0 \\
\hline T19 & 4 & $\mathrm{M}$ & Cerebelar & 2 & 0 & 0 & 2 & 2 & 1 & 0 & 2 \\
\hline T20 & 2 & M & - & 2 & 0 & 0 & 2 & 1 & 1 & 0 & 0 \\
\hline T21 & 37 & $\mathrm{~F}$ & Frontal & 2 & 0 & 0 & 1 & 1 & 2 & 0 & 0 \\
\hline T22 & 33 & M & Ventricular & 3 & 0 & 0 & 2 & 3 & 0 & 1 & 2 \\
\hline T23 & 67 & M & - & 2 & 0 & 1 & 2 & 2 & 0 & 0 & 0 \\
\hline T24 & 23 & $\mathrm{~F}$ & Parietal & 2 & 0 & 0 & 2 & 3 & 0 & 0 & 1 \\
\hline T25 & 48 & M & - & 2 & 0 & 0 & 2 & 3 & 0 & 0 & 1 \\
\hline T26 & 43 & M & - & 2 & 0 & 0 & 2 & 2 & 1 & 0 & 1 \\
\hline T27 & 28 & $\mathrm{~F}$ & - & 3 & 0 & 0 & 2 & 2 & 1 & 0 & 1 \\
\hline T28 & 71 & M & Temporal & 3 & 1 & 0 & 2 & 2 & 2 & 1 & 2 \\
\hline T29 & 56 & M & Parietal & 2 & 0 & 1 & 2 & 3 & 0 & 1 & 1 \\
\hline T30 & 40 & M & Frontal & 3 & 0 & 1 & 2 & 3 & 1 & 1 & 1 \\
\hline T31 & 62 & $\mathrm{~F}$ & Parietal & 3 & 0 & 0 & 2 & 3 & 2 & 0 & 0 \\
\hline T32 & 28 & $\mathrm{~F}$ & Frontal & 2 & 0 & 0 & 1 & 3 & 2 & 0 & 0 \\
\hline T33 & 22 & M & - & 3 & 1 & 0 & 2 & 3 & 2 & 1 & 0 \\
\hline T34 & 27 & $\mathrm{M}$ & - & 3 & 1 & 2 & 2 & 3 & 2 & 0 & 0 \\
\hline T35 & 5 & $\mathrm{~F}$ & - & 3 & 2 & 2 & 3 & 3 & 1 & 1 & 1 \\
\hline T36 & 57 & M & Parietal & 3 & 1 & 1 & 3 & 3 & 3 & 2 & 1 \\
\hline T37 & 43 & $\mathrm{~F}$ & Temporal & 3 & 1 & 2 & 3 & 3 & 3 & 3 & 2 \\
\hline T38 & 62 & M & - & 3 & 1 & 1 & 3 & 3 & 3 & 3 & 1 \\
\hline T39 & 32 & M & - & 3 & 2 & 1 & 3 & 3 & 2 & 3 & 1 \\
\hline T40 & 50 & M & - & 3 & 2 & 0 & 3 & 3 & 3 & 1 & 2 \\
\hline T41 & - & $\mathrm{F}$ & - & 3 & 2 & 0 & 3 & 3 & 2 & 2 & 2 \\
\hline T42 & 34 & M & Frontal & 3 & 2 & 2 & 3 & 3 & 2 & 1 & 2 \\
\hline T43 & 54 & M & - & 3 & 1 & 1 & 3 & 3 & 3 & 3 & 2 \\
\hline T44 & 81 & $\mathrm{~F}$ & Frontal & 3 & 0 & 1 & 3 & 3 & 3 & 1 & 3 \\
\hline T45 & 72 & $\mathrm{~F}$ & - & 3 & 1 & 1 & 3 & 3 & 3 & 1 & 3 \\
\hline T46 & 44 & M & Frontal & 3 & 1 & 1 & 3 & 3 & 3 & 3 & 3 \\
\hline T47 & 62 & $\mathrm{~F}$ & Parietal & 3 & 1 & 1 & 3 & 3 & 3 & 3 & 3 \\
\hline T48 & 43 & M & - & 3 & 2 & 0 & 3 & 3 & 3 & 1 & 3 \\
\hline T49 & 55 & $\mathrm{M}$ & Frontal & 3 & 1 & 1 & 2 & 3 & 1 & 3 & 3 \\
\hline
\end{tabular}




\begin{tabular}{llllclllllllll} 
T50 & IV & 53 & M & Parietal & 3 & 1 & 0 & 3 & 3 & 3 & 3 & 3 & 15 \\
T51 & IV & 50 & M & Parietal & 3 & 2 & 2 & 3 & 3 & 3 & 2 & 3 & 14 \\
T52 & IV & 51 & F & - & 3 & 1 & 1 & 3 & 3 & 3 & 3 & 3 & 15 \\
T53 & IV & 65 & M & Frontal & 3 & 1 & 1 & 3 & 3 & 3 & 3 & 3 & 15 \\
T54 & IV & 55 & M & Occipital & 3 & 1 & 1 & 3 & 3 & 2 & 3 & 2 & 13 \\
T55 & IV & 55 & M & 0ccipital & 2 & 1 & 0 & 3 & 3 & 2 & 2 & 2 & 12 \\
\hline
\end{tabular}

NT: não-tumoral; T: tumoral; Soma: soma dos critérios de graduação histopatológica estabelecidos pela OMS (*)

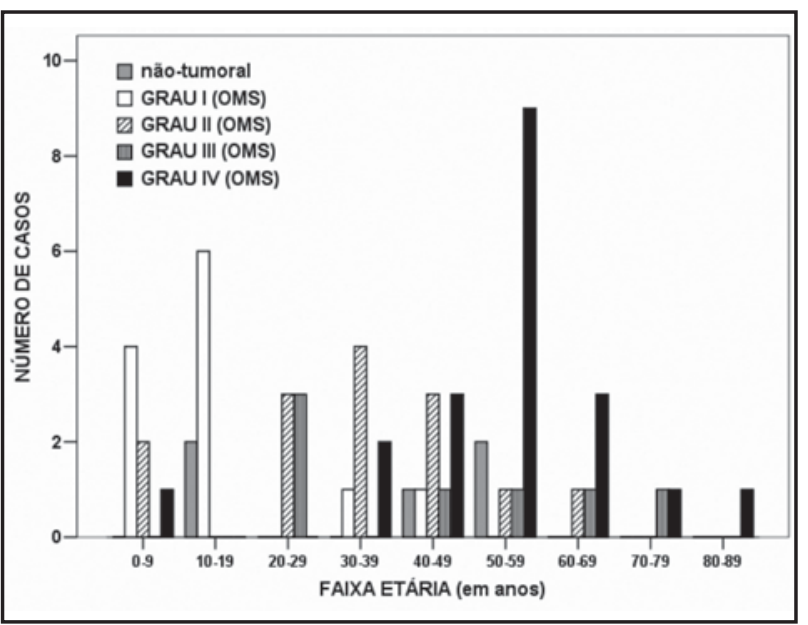

Figura 2 - Distribuição quantitativa dos casos (não-tumorais e astrocitomas) selecionados para o estudo segundo a faixa etária dos pacientes $(n=60)$

\section{Análise histopatológica}

A reavaliação dos achados histopatológicos nos astrocitomas (Figura 3) evidenciou significativos aumentos na celularidade, na atipia nuclear e na presença de necrose conforme a progressão tumoral. A atividade mitótica e a detecção de células gigantes acompanharam a mesma tendência somente nos graus III e IV, enquanto a hiperplasia vascular manifestou-se de forma significativa unicamente nos astrocitomas grau IV. O pleomorfismo celular e a existência de gemistócitos demonstraram propensão ao

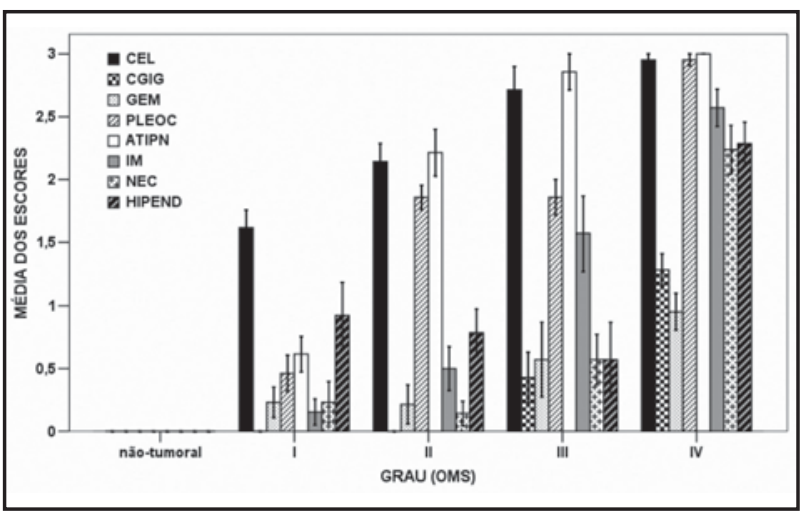

Figura 3 - Médias dos escores atribuídos aos parâmetros histopatológicos segundo a classificação histológica dos casos avaliados; ( $\left(^{*}\right) \mathrm{p}<0,01 ;\left(^{*}\right) \mathrm{p}<0,05$ em relação ao grupo anterior (teste U de Mann-Whitney)

acréscimo, embora não-significativo, de acordo com a gradação tumoral. Nenhuma alteração quanto aos paradigmas avaliados foi constatada nas amostras não-tumorais. A soma dos escores referentes apenas aos critérios de graduação dos astrocitomas adotados pela OMS (Tabela 3) proporcionou clara distinção entre as categorias histológicas.

A análise conjunta de todos os critérios histopatológicos pela metodologia CART (Figura 4) indicou que a simples alteração na celularidade definiu a presença do tumor. $\mathrm{O}$ intenso pleomorfismo celular determinou o grau IV. A associação entre níveis moderados ou intensos de atipia nuclear

Tabela 3

Valores referentes ao somatório dos escores atribuídos aos achados histopatológicos adotados como critérios para graduação tumoral dos astrocitomas pela OMS (pleomorfismo celular, atipia nuclear, indice mitótico, hiperplasia vascular e necrose) segundo a classificação

\begin{tabular}{ccc} 
Tabela 3 & histológica dos casos avaliados \\
\hline Classificação histológica & Média da soma \pm Desvio padrão \\
\hline Não-tumoral & 0 \\
Grau I & $2,38 \pm 1,98^{*}$ \\
Grau II & $5,5 \pm 1,5^{*}$ \\
Grau III & $7,43 \pm 0,97^{*}$ \\
Grau IV & $13,05 \pm 1,56^{*}$ \\
\hline
\end{tabular}

${ }^{*} \mathrm{p}<0,01$ em relação ao grupo anterior (teste U de Mann-Whitney). 


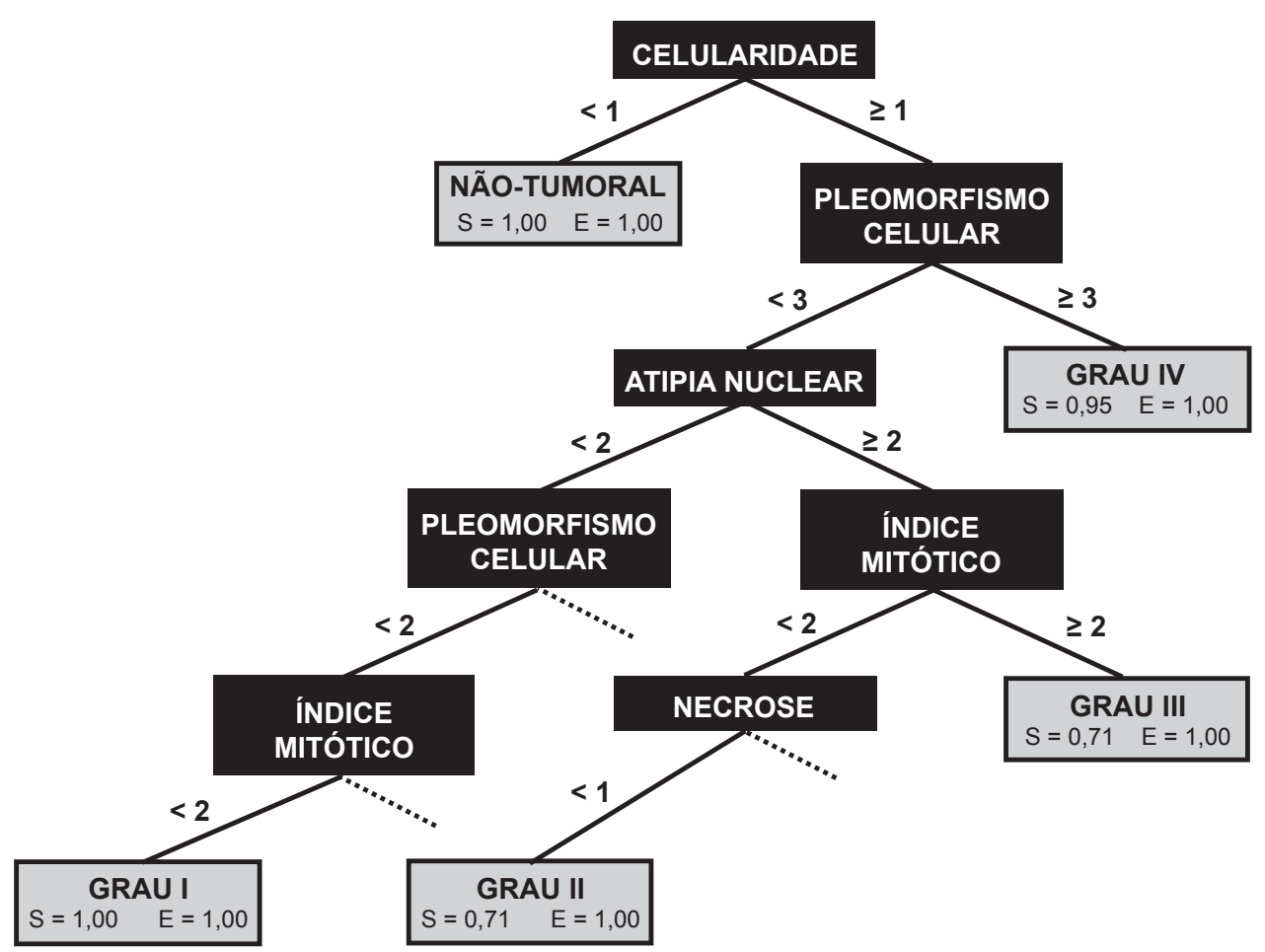

Figura 4 - Árvore de decisão fornecida pelo método CART referente à análise dos achados histopatológicos de acordo com a classificação histológica dos casos avaliados. Custo relativo total: 0,192; S: sensibilidade; E: especificidade.

e de atividade mitótica discriminou os tumores grau III. A conjunção de atipia nuclear moderada ou intensa com figuras mitóticas discretas ou ausentes e com a inexistência de necrose diferenciou o grau II. Os tumores de grau I representaram a combinação entre a discrição ou a ausência dos índices referentes à atipia nuclear, ao pleomorfismo celular e à ocorrência de figuras mitóticas.

\section{Discussão}

\section{Aspectos clínico-epidemiológicos: validando a amostragem}

A distribuição etária dos pacientes da amostra avaliada $(n=55)$ refletiu de modo fidedigno as peculiaridades descritas nos estudos epidemiológicos multicêntricos, com o predomínio dos astrocitomas de baixo grau (I e II) entre os indivíduos mais jovens e dos astrocitomas de alto grau (III e IV) entre os mais idosos (21). A classificação por sexo entre os casos da amostragem revelou a preponderância do masculino, todavia a relação masculino/feminino observada no levantamento geral $(1,28)$ retratou melhor a propensão mundial, estimada em $1,02^{(14)}$.
A localização dos tumores astrocíticos estudados também reproduziu os achados internacionais, com predominância dos tumores grau I na região infratentorial e dos tumores graus II, III e IV em sítios supratentoriais ${ }^{(6,27)}$. Apesar dos astrocitomas grau IV serem descritos mais freqüentemente (31\%) nos lobos temporais ${ }^{(16)}$, cerca da metade dos tumores dessa gradação pertencentes à amostra encontrava-se nos lobos frontais. A despeito do reduzido tamanho amostral, parece razoável supor que a maior porção do tecido cerebral corresponda ao principal local para o surgimento de tumores tipicamente supratentoriais(25). Outra explicação advém da possível extensão de grandes massas tumorais sobre os lobos frontais, caracterizando-as no momento cirúrgico como presentes nessas projeções encefálicas.

\section{Critérios histológicos não considerados pela classificação da OMS}

Embora não figurem entre os critérios de graduação tumoral da OMS, a presença de células gigantes multinucleadas e a detecção de gemistócitos entre os tipos celulares dos tumores astrocíticos são apontadas como indicadores de malignidade ${ }^{(7)}$. Nos astrocitomas investigados, células gigantes foram evidenciadas somente nos tumores de alto grau (III e IV), manifestando-se em associação à elevada celularidade 
e ao expressivo pleomorfismo celular. Diversos autores consideram esses elementos celulares conseqüentes a alterações regressivas (indiferenciação) e sugerem sua correlação com pior prognóstico clínico ${ }^{(14,24)}$. Já os gemistócitos foram encontrados desde os astrocitomas de baixo grau (I e II), todavia com maior representação entre os tumores de alto grau, assim como relatado na literatura(28). Esses componentes celulares, por sua vez, são descritos como possivelmente resultantes da degeneração de astrócitos neoplásicos, conquanto sua origem permaneça incerta ${ }^{(7,18)}$. Tumores astrocíticos de baixo grau, em que são demonstrados gemistócitos, exibem tendência à rápida progressão para subtipos mais malignos. Apesar disso, não se constata correlação direta entre a proporção de gemistócitos e o prognóstico dos pacientes ${ }^{(28)}$.

\section{Análise histopatológica: reconhecendo e elabo- rando estratégias de graduação tumoral}

Qualquer esquema de classificação/graduação fundamentado exclusivamente em parâmetros visuais é, por definição, subjetivo e dá margem a consideráveis variações interobservador. Na prática histodiagnóstica, freqüentemente surgem tumores astrocíticos de complicada classificação/ gradação, precipitando a emissão de laudos dúbios e/ou com diagnósticos imprecisos ${ }^{(19)}$. Além disso, em tumores de difícil diagnóstico, constata-se grande pressão sobre os patologistas, uma vez que o diagnóstico preciso e a classificação acurada permanecem o único meio para a indicação de condutas terapêuticas mais adequadas, assim como para o fornecimento de informações prognósticas $(4,9,15,25)$.

Apesar do grande avanço na uniformização diagnóstica dos gliomas obtido a partir da publicação dos novos critérios da OMS em 1993 e 2000 ${ }^{(15,16)}$, vários estudos apontam a baixa reprodutibilidade e a preocupante variação no diagnóstico e na graduação dos tumores astrocíticos, mesmo quando elaborados por patologistas experimentados $(4,20,30)$. Coons et $a l^{\left({ }^{(8)}\right.}$ evidenciaram que a concordância entre quatro experientes neuropatologistas era de somente $50 \%$ para a classificação e a gradação de astrocitomas, oligodendrogliomas e oligoastrocitomas, aumentando para modestos 70\% após a revisão conjunta dos casos e discussão dos critérios diagnósticos. Gupta et al.(12) ressaltam que essa tendência pode ser facilmente observada pela constatação de marcantes diferenças nas estatísticas diagnósticas quanto aos tipos histológicos e gradações obtidas pelos neuropatologistas de diferentes centros, mesmo quando selecionados aqueles com maior experiência. Esses achados revelam claramente a necessidade do aprimoramento da metodologia de classificação e graduação dos astrocitomas, objetivando maior facilidade e acurácia diagnóstica.

Nesse sentido, o presente estudo averiguou a aplicabilidade de abordagens semiquantitativas (baseadas nos critérios de classificação da OMS) como estratégias auxiliares para graduação dos tumores astrocíticos, bem como o comportamento dessas variáveis na geração de modelos diagnósticos pelo método CART. Esse método foi escolhido por lidar elegante e eficientemente com a tarefa de encontrar modelos representados por árvores de decisão com complexidade limitada(3). Ademais, o CART e outros métodos de inteligência artificial têm sido usados com sucesso na geração de modelos diagnósticos em medicina, notadamente através do uso de sistemas especialistas dedutivos e na indução de modelos de conhecimento a partir de uma coleção retrospectiva de $\operatorname{casos}^{(6,11,17)}$.

A reavaliação dos achados histopatológicos nos astrocitomas pesquisados constatou que a alteração da celularidade define a presença de neoplasia; a atipia nuclear caracteriza os tumores de grau II, enquanto sua associação à atividade mitótica distingue os tumores de grau III; a presença de hiperplasia endotelial e/ou necrose diferencia os tumores de grau IV em relação ao grau III; discretas alterações histológicas discriminam os tumores de grau I. Esses achados coincidem integralmente com os critérios estabelecidos pela OMS para graduação dos tumores astrocíticos $^{(15)}$, confirmando o diagnóstico original e reiterando a aplicabilidade e a reprodutibilidade dos mesmos.

Ressalta-se, no entanto, que tais resultados foram obtidos através não da usual análise qualitativa de achados histológicos, mas da atribuição de escores semiquantitativos propostos pelo presente estudo (Tabela 1) de acordo com os paradigmas preconizados pela OMS para a classificação dos astrocitomas. Tanto as variações individuais como o somatório das pontuações demonstraram valor preditivo em relação à gradação tumoral. Dessa forma, percebe-se a adequação dessa estratégia como auxiliar na determinação do grau dos tumores astrocíticos, tendo os valores médios da soma dos escores analisados (Tabela 3) como principal referência para a classificação dessas neoplasias.

Outro importante instrumento para distinção da graduação dos astrocitomas surgiu com a aplicação da metodologia CART (Figura 4). Além da combinação dos critérios já descritos, a árvore de decisão referente à análise histopatológica dos tumores astrocíticos apontou o pleomorfismo celular intenso como marca registrada do grau IV. De fato, poucos neoplasmas humanos demonstram tamanha heterogeneidade em sua composição celular como os astrocitomas grau $\operatorname{IV}^{(10,14,16,24)}$. Nesses tumores, transições entre áreas 
com diferenciação astrocítica discernível e regiões altamente anaplásicas mostram-se freqüentes e, por vezes, ocorrem de forma abrupta. Essas variações súbitas parecem indicar o aparecimento de novos fenótipos tumorais desencadeados pela aquisição de alterações genéticas diversas ${ }^{(18,23)}$.

A elaboração/formatação de árvores de decisão motivadas pela necessidade de maior objetividade e acurácia na rotina histodiagnóstica apresenta algumas vantagens, a citar o alto índice de precisão dos modelos obtidos (elevada acurácia diagnóstica), a exibição gráfica do modelo através da estrutura de uma árvore de decisão binária (baixa complexidade), a possibilidade de formular regras de associação que poderão ser facilmente compreendidas pelos usuários (elevado grau de interpretabilidade), a habilidade para lidar com grandes bases de dados contendo variáveis de diversas naturezas (nominal, ordinal e contínua), a análise de variáveis sem necessidade de constantes recodificações e a freqüente descoberta de informações novas, inesperadas ${ }^{(11,13)}$.

Salienta-se, entretanto, que a aplicabilidade dos métodos propostos limita-se aos tipos tumorais primariamente enumerados na amostragem do presente estudo, uma vez que os testes estatísticos e a análise CART não podem, obviamente, versar sobre dados não listados originalmente. Com isso, entidades tumorais raras e atípicas não avaliadas, a exemplo do xantoastrocitoma pleomórfico, do astrocitoma subependimário de células gigantes ou mesmo do astrocitoma desmoplásico infantil, possivelmente não admitiriam os critérios expostos nos escores/médias e na árvore de decisão CART para gradação dos astrocitomas. Diz-se, então, que o modelo obtido excede o conjunto de casos (overfit). Isso significa que o modelo consegue diagnosticar corretamente quase todos os casos novos idênticos aos usados para gerálo, porém, é impreciso quando se trata de diagnosticar novos casos desconhecidos. Assim, devem-se buscar classificadores que sejam menos ligados à casuística disponível, porém, mais gerais, que consigam definir corretamente o diagnóstico de novos casos. Um modelo mais geral é menos complexo no que diz respeito ao seu tamanho (rightfit). Em resumo, busca-se um bom compromisso entre complexidade e número de diagnósticos corretos, considerando-se apenas os casos disponíveis que alimentaram o sistema ${ }^{(5,17)}$.

A categorização é algo necessário na prática médica, mas não deve ser encarada como fundamento estático ou impeditivo de progressos. Parece improvável que a classificação/ graduação dos tumores astrocíticos permaneça inalterada diante dos fascinantes avanços advindos dos recentes estudos moleculares. Todavia, as evidências históricas sugerem que a abordagem molecular dos tumores dificilmente irá suplantar o estudo histológico completamente, pelo menos num futuro próximo. Análises ultra-estruturais e imuno-histoquímicas forneceram importantes evidências para o aprimoramento das classificações histopatológicas, mas também não foram capazes de superar a avaliação por microscopia óptica como ferramenta diagnóstica primária ${ }^{(15,16,19,23)}$.

Diante do exposto, acredita-se que o uso dos escores/ médias e da árvore de decisão para gradação dos tumores astrocíticos apresentados neste estudo configurem artifícios aplicáveis na prática diagnóstica como auxiliares na determinação do grau histológico dos astrocitomas, especialmente os de difícil categorização.

\section{Agradecimentos}

Ao Professor-doutor Fernando de Carvalho Gomes, pesquisador do Laboratório de Inteligência Artificial do Departamento de Computação da UFC, pelo suporte na aplicação dos métodos de aprendizagem automática utilizados neste ensaio.

\section{Referências}

I. BAILEY, P.; CUSHING, H. A classification of tumors of the gliomas group on a histogenetic basis with a correlated study of prognosis. Philadelphia: Lippincott, 1926.

2. BREIMAN, L. et al. Classification and regression trees. Monterey: Wadsworth, 1984

3. BRUNER, J. M. et al. Diagnostic discrepancies and their clinical impact in a neuropathology referral practice. Cancer, v. 79 , p. 796-803, 1997.

4. BUCKNER, J. C. Factors influencing survival in high-grade gliomas. Semin Oncol, v. 30, n. 6, suppl. 19, p. 10-4, 2003.

5. CARVALHO-GOMES, F.; GASCUEL, O. SDL, a stochastic algorithm for learning decision lists with limited complexity. Ann Mathem Artif Intelig, v. 10, p. 281-302, 1994.

6. CBTRUS. Statistical report: primary brain tumours in the United States (1997-200 I). Chicago: Central Brain Tumor Registry of the United States, p. I5-8, 2004.

7. COLLINS, V. P. Brain tumours: classification and genes. J Neurol Neurosurg Psychiatry, v. 75, suppl. II, p. ii2-iil I , 2004.

8. COONS, S. W. et al. Improving diagnostic accuracy and interobserver concordance in the classification and grading of primary gliomas. Cancer, v. 79, p. I 381-93, 1997.

9. DYBOWSKI, R.; GANT,V. Artificial neural networks in pathology 
and medical laboratories. Lancet, v. 346, p. 1203-7, 1995.

I0. FARIA, M. H. G.; PATROCÍNIO, R. M. S.V.; RABENHORST, S. H. B. Astrocitomas: uma revisão abrangente. Rev Bras Neurocir, v. 25, n. I, p. 23-33, 2006.

I I. GUDINAVI IENË, I.; PRANYS, D.; JUOZAITYTË, E. Impact of morphology and biology on the prognosis of patients with gliomas. Medicina, v. 40, n. 2, p. I I 2-20, 2004.

12. GUPTA, M.; DJALILVAND, A.; BRAT, D. J. Clarifying the diffuse gliomas: an update on the morphologic features and markers that discriminate oligodendroglioma from astrocytoma. Am J Clin Pathol, v. I24, n. 5, p. 755-68, 2005.

13. HILTON, D.A.; MELLING, C. Genetic markers in the assessment of intrinsic brain tumors. Curr Diagn Pathol, v. 10. p. 83-92, 2004.

14. KLEIHUES, P. et al. Pathology and genetics of tumours of the central nervous system. In: KLEIHUES, P.; CAVENEE, W. K. (eds.) World Health Organization Classification of Tumours. Lyon: IARC Press, 2000. p. 6-69.

15. KLEIHUES, P. et al.The WHO classification of tumours of the nervous system. J Neuropathol Exp Neurol, v. 6I, n. 3, p. 215-25, 2002.

16. LANTOS, P. L.; ROSENBLUM, M. K.; KLEIHUES, P.Tumours of the nervous system. In: GRAHAN, D. I.; LANTOS, P. L. (eds.) Greenfield's Neuropathology vol. 2. $7^{\text {th }}$ ed. Glasgow: Arnold Publishers, 2002. p. 767- 1052

17. LEITÃO, F. et al. Diagnóstico de tumores do ângulo pontocerebelar com o auxilio de técnicas de inteligência artificial. Arq Neuropsiquiatr, v. 58, n. I, p. 57-63, 2000.
I8. LOUIS, D. N.; POMEROY,S. L.; CAIRNCROSS,J.G. Focus on central nervous system neoplasia. Cancer Cell, v. I, p. I25-8, 2002.

19. LOUIS, D. N.; HOLLAND, E. C.; CAIRNCROSS, J. G. Glioma classification: a molecular reappraisal. Am J Pathol, v. I59, n. 3, p. 779-86, 200।.

20. MITTLER, M.;WALTERS, B. C.; STOPA, E. G. Observer reliability in histological grading of astrocytoma stereotactic biopsies. J Neurosurg, v. 85, p. I091-4, 1996.

2I.PEKMEZOVIC,T.; JAREBINSKI, M.; PAVLOVIC, M. Epidemiology of central nervous system tumours. Arch Oncol, v. 10, n. 3, p. 177-8, 2002.

22. PRAYSON, R. A. et al. Interobserver reproducibility among neuropathologists and surgical pathologists in fibrillary astrocytoma grading. J Neurol Sci, v. I75, n. I, p. 33-9, 2000.

23. REIFENBERGER, G.; COLLINS, V. P. Pathology and molecular genetics of astrocytic gliomas. J Mol Med, v. 82, n. 10, p. 656-70, 2004

24. SCHMIDT, M. C. et al. Impact of genotype and morphology on the prognosis of glioblastoma. J Neuropathol Exp Neurol, v. 61, n. 4, p. 321-8, 2002.

25.TAILLIBERT, S.; PEDRETTI, M.;SANSON, M. Current classification of gliomas. Presse Med, v. 33, n. 18, p. 1274-7, 2004.

26.VIRCHOW, R. Die krankhaften geschwülste. Berlin: Hirshwald, 1863.

27. WALKER, D. G.; KAYE, A. H. Diagnosis and management of astrocytomas, oligodendrogliomas and mixed gliomas: a review. Austral Radiol, v. 45, p. 472-82, 200 I.

28.YANG, H. J. et al.The significance of gemistocytes in astrocytoma. Acta Neurochir (Wien), v. I45, n. I2, p. I097- 103, 2003. 